\title{
Editorial
}

\section{Differential Geometry: An Introduction to the Theory of Curves}

\author{
Kande Dickson Kinyua ${ }^{1,2}$, Kuria Joseph Gikonyo ${ }^{2}$ \\ ${ }^{1}$ Department of Mathematics, Moi University, Eldoret, Kenya \\ ${ }^{2}$ Department of Mathematics, Karatina University, Karatina, Kenya \\ Email address: \\ kandesnr@gmail.com (K. D. Kinyua),josephgkuria@gmail.com (K. J. Gikonyo)
}

To cite this article:

Kande Dickson Kinyua, Kuria Joseph Gikonyo. Differential Geometry: An Introduction to the Theory of Curves. International Journal of Theoretical and Applied Mathematics. Vol. 3, No. 6, 2017, pp. 225-228. doi: 10.11648/j.ijtam.20170306.18

Received: December 4, 2016; Accepted: January 18, 2017; Published: January 10, 2018

\begin{abstract}
Differential geometry is a discipline of mathematics that uses the techniques of calculus and linear algebra to study problems in geometry. The theory of plane, curves and surfaces in the Euclidean space formed the basis for development of differential geometry during the 18th and the 19th century. The core idea of both differential geometry and modern geometrical dynamics lies under the concept of manifold. A manifold is an abstract mathematical space, which locally resembles the spaces described by Euclidean geometry, but which globally may have a more complicated structure. The purpose of this paper is to give an elaborate introduction to the theory of curves, and those are, in general, curved. Differential geometry of curves is the branch of geometry that deals with smooth curves in the plane and in the Euclidean space by applying the concept of differential and integral calculus. The curves are represented in parametrized form and then their geometric properties and various quantities associated with them, such as curvature and arc length expressed via derivatives and integrals using the idea of vector calculus.
\end{abstract}

Keywords: Curvature, Curves, Differential Geometry, Manifolds, Parametrized

\section{Introduction}

Differential geometry is a discipline of mathematics that uses the techniques of calculus and linear algebra to study problems in geometry. The theory of plane, curves and surfaces in the three-dimensional Euclidean space formed the basis for development of differential geometry during the 18 th and the 19 th century. Since the late 19th century, differential geometry has grown into a field concerned more generally with the geometric structures on differentiable manifolds, [2, 4, 6] Differential geometry is closely related to differential topology and the geometric aspects of the theory of differential equations. Differential geometry arose and developed as a result of and in connection to the mathematical analysis of curves and surfaces. The theory developed in this study originates from mathematicians of the 18th and 19th centuries, mainly; Euler (1707-1783), Monge (1746-1818) and Gauss (1777-1855). Mathematical study of curves and surfaces has been developed to answer some of the nagging and unanswered questions that appeared in calculus, such as the reasons for relationships between complex shapes and curves, series and analytic functions. The purpose of this paper is to give an elaborate introduction to the theory of curves, and those are, in general, curved, $[6,8]$.

Nevertheless, our main tools to understand and analyze these curved objects are (tangent) lines and planes and the way those change along a curve, respective surface. This is why we start with a brief chapter assembling prerequisites from linear geometry and algebra. The objects that will be studied here are curves in two- and three-dimensional space, and they are primarily studied by means of parametrization. The main properties of these objects, which will be studied, are notions related to the shape. The study tangents of curves, and the concept of curvature will be introduced and 
defined through differentiation of the parametrization, and related to first and second derivatives, respectively. The culmination is a famous theorem of Gauss, which shows that the so-called Gauss curvature of a surface can be calculated directly from quantities which can be measured on the surface itself, without any reference to the surrounding three dimensional space, $[5,10]$.

\section{Manifolds}

The core idea of both differential geometry and modern geometrical dynamics lies under the concept of manifold. A manifold is an abstract mathematical space, which locally resembles the spaces described by Euclidean geometry, but which globally may have a more complicated structure, $[6,9]$. A manifold can be constructed by 'gluing' separate Euclidean spaces together; for example, a world map can be made by gluing many maps of local regions together, and accounting for the resulting distortions. Therefore, the surface of Earth is a manifold; locally it seems to be flat, but viewed as a whole from the outer space (globally) it is actually round. Another example of a manifold is a circle; small piece of a circle appears to be like a slightly bent part of a straight line segment, but overall the circle and the segment are different one-dimensional manifolds, [2, 9].

Definition 2.1: A manifold is a Hausdorff space $M$ with a countable basis such that for each point $p \in M$ there is a

neighborhood $\mathrm{U}$ of $\mathrm{p}$ that is homeomorphic to $R^{n}$ for some integer $\mathrm{n}$. If the integer $\mathrm{n}$ is the same for every point in $M$, then $\mathrm{M}$ is called a n-dimensional manifold, $[1,2,9]$.

Definition 2.2: A topological space $\mathrm{X}$ is said to be Hausdorff if for any two distinct points $x, y \in X$ there exist disjoint open sets $U$ and $\mathrm{V}$ with $x \in U$ and $y \in V,[1,9]$.

The study of manifolds combines many important areas of mathematics: it generalizes concepts such as curves with the ideas from linear algebra and topology. Certain special classes of manifolds also have additional algebraic structure; they may behave like groups, for instance. From $[1,4,5]$, an atlas describes how a manifold is glued together from simpler pieces where each piece is given by a chart (coordinate chart or local coordinate system). The description of most manifolds requires more than one chart. An atlas is a specific collection of charts which covers a manifold. An atlas is not unique as all manifolds can be covered multiple ways using different combinations of charts, [4, 5, 8].

Definition 2.3: An atlas $A$ on a manifold $M$ is said to be maximal if for any compatible atlas $A^{\prime}$ on $M$ any coordinate chart $(x, U) \in A^{\prime}$ is also a member of $A,[1,9]$.

This definition of atlas is exactly analogous to the nonmathematical meaning of atlas. Each individual map in an atlas of the world gives a neighborhood of each point on the globe that is homeomorphic to the plane. While each individual map does not exactly line up with other maps that it overlaps with, the overlap of two maps can still be compared. Different choices for simple spaces and compatibility conditions give different objects. The dimension of the manifold at a certain point is the dimension of the Euclidean space charts at that point map to (number $n$ in the definition), [2], [3], [9]. All points in a connected manifold have the same dimension. In topology and related branches of mathematics, a connected space is a topological space which cannot be written as the disjoint union of two or more nonempty spaces. Connectedness is one of the principal topological properties that is used to distinguish topological spaces. A manifold with empty boundary is said to be closed manifold if it is compact, and open manifold if it is not compact. All one-dimensional manifolds are curves and all two-dimensional manifolds are surfaces, [1, 3, 9].

\section{Curves}

Differential geometry of curves is the branch of geometry that deals with smooth curves in the plane and in the Euclidean space by applying the concept of differential and integral calculus. The curves are represented in parametrized form and then their geometric properties and various quantities associated with them, such as curvature and arc length expressed via derivatives and integrals using the idea of vector calculus, $[4,5,7]$.

One of the most important tools used to analyze a curve is the Frenet frame, which is a moving frame that provides a coordinate system at each point of the curve that is "best adapted" to the curve near that point. Different space curves are only distinguished by the way in which they bend and twist and quantitatively measured by the differentialgeometric invariants called curvature and torsion of the curve. The fundamental theorem of curves asserts that the knowledge of these invariants completely determines the curve, $[4,5,7]$.

Definition 3.1: For a particle moving in space, at any time $t$, its position is given by, $[4,5,7]$.

$$
\alpha(t)=(x(t), y(t), z(t)) \in R^{3}
$$

Definition 3.2: Definition of parameterized differentiable curve

A parameterized differentiable curve is a differentiable map $\alpha: I \rightarrow R^{3}$ of an open interval $I=(a, b)$ of the real line $R$ into $R^{3} \alpha$ maps $t \in I$ into a point

$$
\alpha(t)=(x(t), y(t), z(t)) \in R^{3}
$$

such that $x(t), y(t), z(t)$ are differentiable, $[4,5,7]$.

The term differentiable means that $\alpha$ is a correspondence which maps each $t \in I$ into equation (2) in such a way that the functions $x(t), y(t), z(t)$ are differentiable. The variable is called the parameter of the curve, $[4,5,7]$.

Definition 3.3: Definition of tangent vector

Let $\alpha: I \rightarrow R^{3}$ be a parameterized differentiable curve. For each $t \in I$ where $\alpha^{\prime}(t) \neq 0$, there is a well defined straight line which contains the point $\alpha(t)$ and the vector 
$\alpha^{\prime}(t)$; this line is called tangent line to $\alpha$ at $\mathrm{t}$.

If $x^{\prime}(t)$ denotes the first derivative of $\mathrm{x}$ at point $\mathrm{t}$, then $\mathrm{a}$ similar notation for the function $\mathrm{y}$ and $\mathrm{z}$, the vector

$$
\left(x^{\prime}(t), y^{\prime}(t), z^{\prime}(t)\right)=\alpha^{\prime}(t) \in R^{3}
$$

is called the tangent vector of the curve $\alpha$ at t. if this tangent vector is a unit vector, it is refereed as the unit tangent vector, $T(s),[4,5,7]$.

Definition 3.4: Let $\alpha: I \rightarrow R^{3}$ be a curve. The unit vectors $T(s), N(s), B(s)$ are called unit-tangent, principal normal and binormal vectors of $\alpha$ respectively. They are related in the following manner, $[4,5,7]$;

$$
\left.\begin{array}{l}
B(s)=T(s) \times N(s) \\
N(s)=B(s) \times T(s) \\
T(s)=N(s) \times B(s)
\end{array}\right\}
$$

\section{Definition 3.5: Definition of arc-length}

A parameterized differentiable curve $\alpha: I \rightarrow R^{3}$ is said to be regular if $\alpha^{\prime}(t) \neq 0$ for all $t_{0}$.

The arc-length of a regular parameterized differentiable curve $\alpha: I \rightarrow R^{3}$ from the point $t_{0}$ is defined by,

$$
\left|\alpha^{\prime \prime}(t)\right|=\kappa(s)
$$

is called the arc-length, $[4,5,7]$.

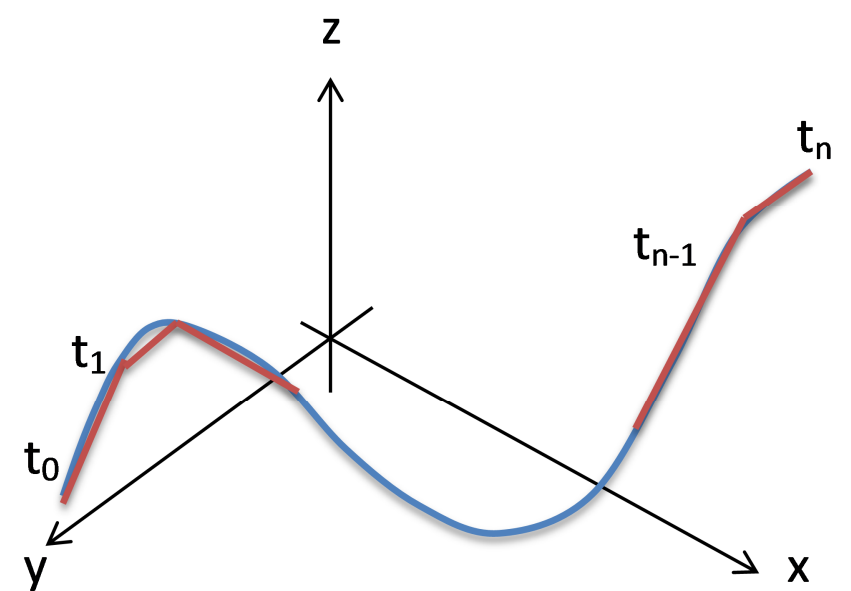

Figure 1. Arc-length can be determined by partitioning $\left(t_{0}, t\right)$ into $n$ subintervals with $t_{i}=t_{0}+i \Delta t$ then sum the integrals for the arc-length.

\section{Definition 3.6: Curvature}

Let $\alpha: I \rightarrow R^{3}$ be a curve parameterized by arc length s. The curvature of $\alpha$ at $\mathrm{s}$ is defined by:

$$
\left|\alpha^{\prime \prime}(t)\right|=\kappa(s)
$$

where,

$\alpha^{\prime}(\mathrm{s})$ - the tangent vector at $\mathrm{s}$

$\alpha^{\prime \prime}(s)$ - the change in the tangent vector at $s$
$R(s)=\frac{1}{\kappa(s)}$ is called the radius of curvature at $\mathrm{s}$.

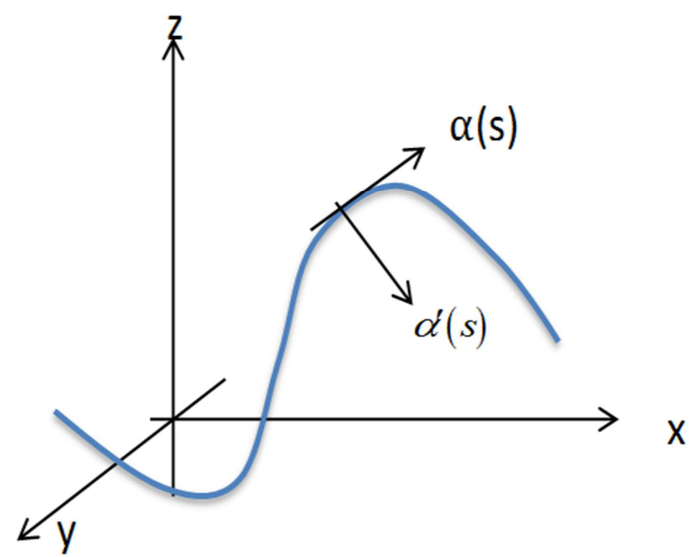

Figure 2. At points where $\kappa(s) \neq 0$ in the direction $\alpha^{\prime \prime}(s)$, there exist a unit vector $N(s)$ called the normal vector.

The normal vector is defined by,

$$
\alpha^{\prime \prime}(s)=\kappa(s) N(s)
$$

Since $\alpha^{\prime}(s)$ and $\alpha^{\prime \prime}(s)$ are perpendicular,

$$
\alpha^{\prime \prime}(s) \bullet \alpha^{\prime}(s)=0
$$

The plane determined by the unit tangent and normal vectors $\mathrm{T}(\mathrm{s})$ and $\mathrm{N}(\mathrm{s})$ is called the osculating plane at $\mathrm{s},[4,5,7]$.

\section{Osculating plane}

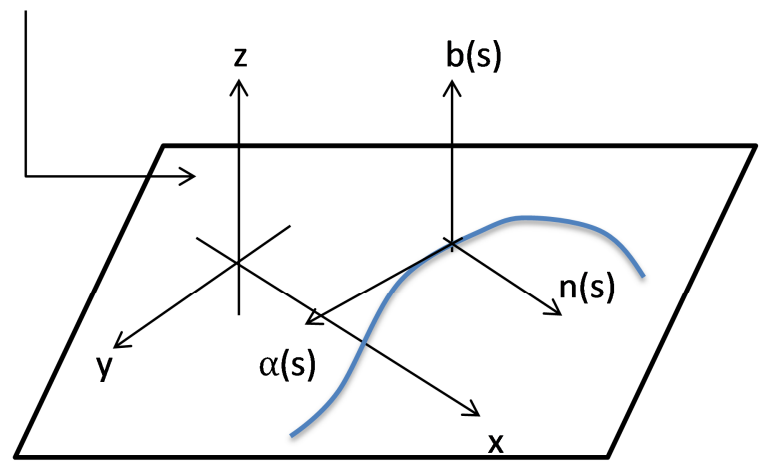

Figure 3. The osculating plane to a curve.

\section{Definition 3.7: Torsion}

Let $\alpha: I \rightarrow R^{3}$ be a curve parameterized by arc length s. The binormal vector is always orthogonal to the osculating plane; therefore, derivative of the binormal vector with respect to arc-length measures how fast the osculating plane changes as s varies. Thus,

$$
B^{\prime}(s)=-\tau(s) N(s)
$$

Definition 3.8: The Frenet-Serret formulas

Given differentiable functions $\kappa(s)>0$ and $\tau(s), s \in I$, there exists a regular parameterized curve $\alpha: I \rightarrow R^{3}$ such 
that $s$ is the arc length, $\kappa(s)$ is the curvature, and $\tau(s)$ is the torsion of $\alpha$. Moreover, any other curve $\bar{\alpha}$, satisfying the same conditions differs from $\alpha$ only by a rigid motion. The three vectors $T(s), N(s), B(s)$ constitute a positively ordered orthonormal basis for $R^{3}$ (depending on s), which is called the moving frame of Frenet for the curve or the Frenet (or of Frenet-Serret) formulas. The Frenet-Serret formulas are used to describe the kinetic properties of a particle moving along a continuous, differentiable curve in $R^{3}$, or the geometric properties of the curve itself irrespective of any motion. These formulas are, $[4,5,7]$;

$$
\left.\begin{array}{l}
\text { i) } T^{\prime}(s)=\kappa(s) N(s) \\
\text { ii) } N^{\prime}(s)=-\kappa(s) T(s)+\tau(s) B(s) \\
\text { iii) } B^{\prime}(s)=-\tau(s) N(s)
\end{array}\right\}
$$

Proof:

a) Show that, $T^{\prime}(s)=\kappa(s) N(s)$

By equation (6), $T^{\prime}(s)=\kappa(s)$ so $T^{\prime}(s)$ will have the same magnitude as $\kappa(s) N(s)$. By definition, $N(s)=\frac{T^{\prime}(s)}{\left|T^{\prime}(s)\right|}$, but the parametrization used only affects the $N(s)$ by a scalar factor of \pm 1 . Hence $N(s)$ has the same direction as $T^{\prime}(s)$ and by definition $\kappa \geq 0$. Thus, $T^{\prime}(s)$ and $\kappa(s) N(s)$ have the same direction and magnitude, so they are equal.

b) Show that, $B^{\prime}(s)=-\tau(s) N(s)$

Since $B^{\prime}(s)$ is perpendicular to both $B(s)$ and $T(s)$, it must be parallel to $N(s)$. We define $-\tau(s)$ to be the scaling factor such that $B^{\prime}(s)=-\tau(s) N(s)$.

c) Show that, $N^{\prime}(s)=-\kappa(s) T(s)+\tau(s) B(s)$

From (a) and (b) above, $T^{\prime}(s)=\kappa(s) N(s)$ and $B^{\prime}(s)=-\tau(s) N(s)$ while from equation (4),

$$
\begin{aligned}
N(s) & =B(s) \times T(s) \Leftrightarrow N^{\prime}(s)=(B(s) \times T(s))^{\prime} \\
N^{\prime}(s) & =(B(s) \times T(s))^{\prime} \\
& =B^{\prime}(s) \times T(s)+B(s) \times T^{\prime}(s) \\
& =\tau(\mathrm{s})[N(s)] \times T(s)+B(s) \times \kappa(s)[N(s)] \\
& =\tau(\mathrm{s})[T(s) \times N(s)]-\kappa(s)[N(s) \times B(s)] \\
& =\tau(\mathrm{s}) \mathrm{B}(\mathrm{s})-\kappa(s) T(s)
\end{aligned}
$$

Since each of the functions $T(s), N(s), B(s)$ have three coordinates, this is essentially a linear system of nine first order differential equations in these coordinates; by solving this system one can (at least in principle) determine a curve from its curvature $\kappa(s)$ and torsion $\tau(s)$, up to integration constants. To each value of the parameter $\mathrm{s}$, the three associated orthogonal unit vectors $T(s), N(s), B(s)$ form a trihedron known as the Frenet frame, [4, 5, 7].

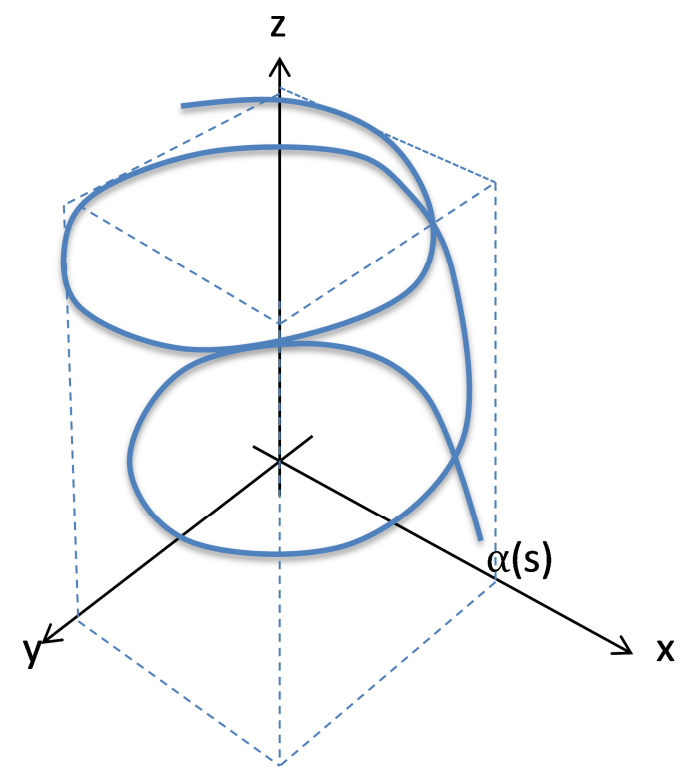

Figure 4. The Frenet trihedron or the Frenet frame.

\section{Conclusion}

For any regular curve parametrized by arc length in three dimensions is defined easily by considering a special moving frame, called standard Frenet-Serret frame that satisfy the Frenet equations. As the Frenet-Serret frame in space, the curvature and the torsion of the curve also change considerably with respect to the curve equation.

\section{References}

[1] K. D. Kinyua. An Introduction to Differentiable Manifolds, Mathematics Letters. Vol. 2, No. 5, 2016, pp. 32-35.

[2] Lang, Serge, Introduction to Differentiable Manifolds, 2nd ed. Springer-Verlag New York. ISBN 0-387-95477-5, 2002.

[3] M. Deserno, Notes on Difierential Geometry with special emphasis on surfaces in $\mathrm{R}^{3}$, Los Angeles, USA, 2004.

[4] M. P. do-Carmo, Differential Geometry of curves and surfaces, Prentice-Hall, Inc., Englewood Cliffs, New Zealand, USA, 1976.

[5] M. Raussen, Elementary Differential Geometry: Curves and Surfaces, Aalborg University, Denmark, 2008.

[6] M. Spivak, A Comprehensive Introduction to Differential Geometry, Vol. 1, Third Edition, Publish or Perish Inc., Houston, USA, 1999.

[7] R. Palais, A Modern Course on Curves and Surfaces, 2003.

[8] T. Shifrin, Differential Geometry: A First Course in Curves and Surfaces, Preliminary Version, University of Georgia, 2016.

[9] V. G. Ivancevic and T. T. Ivancevic Applied Differential Geometry: A Modern Introduction, World Scientific Publishing Co. Pte. Ltd., Toh Tuck Link, Singapore, 2007.

[10] W. Zhang, Geometry of Curves and Surfaces, Mathematics Institute, University of Warwick, 2014. 